\title{
Clinicopathological factors associated with HER2 status in gastric cancer: results from a prospective multicenter observational cohort study in a Japanese population (JFMC44-1101)
}

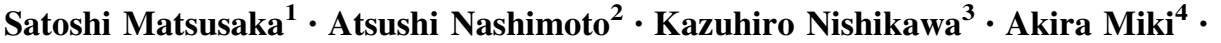 \\ Hiroto Miwa ${ }^{5}$ Kazuya Yamaguchi ${ }^{6}$ - Takaki Yoshikawa ${ }^{7} \cdot$ Atsushi Ochiai $^{8}$. \\ Satoshi Morita ${ }^{9}$ Takeshi Sano $^{1}$ - Yasuhiro Kodera ${ }^{10}$ - Yoshihiro Kakeji ${ }^{11}$ • \\ Junichi Sakamoto $^{12} \cdot$ Shigetoyo Saji $^{12} \cdot$ Kazuhiro Yoshida ${ }^{6}$
}

Received: 19 March 2015/Accepted: 10 July 2015/Published online: 12 August 2015

(C) The Author(s) 2015. This article is published with open access at Springerlink.com

\begin{abstract}
Background Human epidermal growth factor (HER) 2 positivity and its association with clinicopathological factors remain unclear in Japanese gastric cancer (GC) patients. We performed a prospective, multicenter, observational cohort study to evaluate HER2 protein expression and gene amplification in Japanese metastatic and recurrent GC patients, and explored its correlations with clinicopathological features.

Methods HER2 protein expression and gene amplification were centrally assessed in formalin-fixed, paraffinembedded GC tissue by immunohistochemistry (IHC) and fluorescence in situ hybridization (FISH). Patient information was collected, and associations between clinicopathological factors and HER2 positivity (IHC score $3+$ and/or FISH positive) and low HER2 expression (IHC score 0/FISH positive or IHC score $1+$ /FISH positive) were examined.
\end{abstract}

\section{Kazuhiro Yoshida}

kyoshida@gifu-u.ac.jp

1 Cancer Institute Hospital of the Japanese Foundation for Cancer Research, Tokyo, Japan

2 Department of Surgery, Niigata Prefectural Cancer Center Niigata Hospital, Niigata, Japan

3 Department of Surgery, Osaka General Medical Center, Osaka, Japan

4 Department of Surgery, Kobe City Medical Center General Hospital, Kobe, Japan

5 Division of Gastroenterology, Department of Internal Medicine, Hyogo College of Medicine, Nishinomiya, Japan

6 Department of Surgical Oncology, Gifu University Graduate School of Medicine, 1-1 Yanagido, Gifu 501-1194, Japan
Results From September 2011 to June 2012, 1461 patients were registered across 157 sites, and the HER2 status of 1427 patients was evaluated. The rate of HER2 positivity was $21.2 \%$, whereas the rate of high HER2 expression (IHC score 2+/FISH positive or IHC score $3+$ ) was $15.6 \%$ and that of low HER2 expression was $7.0 \%$. Multiple logistic regression analysis identified intestinal type, absence of peritoneal metastasis, and hepatic metastasis as significant independent factors related to HER2 positivity. The intestinal type was confirmed to be the GC subtype predominantly associated with lower HER2 expression. Sampling conditions including number of biopsy samples, formalin concentration, and formalin-fixation time did not significantly affect HER2 positivity.

Conclusions HER2 expression in Japanese patients was comparable to that in other populations examined. Intestinal type was an independent factor related to HER2 positivity and low HER2 expression.

7 Department of Gastrointestinal Surgery, Kanagawa Cancer Center, Yokohama, Japan

8 Research Center for Innovative Oncology, National Cancer Center Hospital East, Chiba, Japan

9 Kyoto University Graduate School of Medicine, Kyoto, Japan

10 Department of Gastroenterological Surgery (Surgery II), Nagoya University Graduate School of Medicine, Nagoya, Japan

11 Division of Gastrointestinal Surgery, Department of Surgery, Graduate School of Medicine, Kobe University, Kobe, Japan

12 Japanese Foundation for Multidisciplinary Treatment of Cancer, Tokyo, Japan 
Keywords Fluorescence in situ hybridization - Stomach neoplasms · Human ERBB2 protein .

Immunohistochemistry

\section{Introduction}

Trastuzumab (Herceptin) is a monoclonal antibody that specifically targets human epidermal growth factor receptor 2 (HER2), a receptor associated with gastric cancer (GC) tumorigenesis, by directly binding its extracellular domain [1]. The Trastuzumab for GAstric Cancer (ToGA) study, an open-label, international, multicenter, phase III, randomized controlled trial, examined the clinical efficacy and safety of trastuzumab combined with standard chemotherapy (capecitabine or intravenously administered 5-fluorouracil and cisplatin) for first-line treatment of HER2overexpressing advanced gastric or gastroesophageal junction cancers. Addition of trastuzumab therapy to chemotherapy improved median survival (13.8 months) compared with chemotherapy alone (11.1 months) $(P=0.0046)$, and showed significant improvements in time to progression and progression-free survival in the trastuzumab-treated group, with a comparable toxicity profile [2]. As a result, trastuzumab therapy plus chemotherapy has become the standard treatment for HER2-positive advanced GC patients, as determined by immunohistochemistry (IHC) and/or fluorescence in situ hybridization (FISH). In Japan and the USA, trastuzumab is approved for patients with metastatic GC whose tumors are HER2 positive, as defined by a positive FISH result or an IHC score of $3+$. In the European Union, however, trastuzumab is recommended only for individuals whose tumors have high HER2 protein expression, as defined by an IHC score of 2+/positive FISH result or an IHC score of $3+$ based on the subset analysis of the ToGA study. HER2 evaluation has therefore become an important approach for predicting clinical efficacy of trastuzumab. The variation in the HER2-positivity rate between countries possibly reflects the unstandardized testing modality and other countryspecific factors; it was identified as $27 \%$ in Japanese patients in the ToGA study $[3,4]$, which was higher than that identified in previous studies in Japan [5-7].

In the ToGA study, the strong effect of trastuzumab was evident in patients with higher HER2 protein expression (IHC score $2+/$ FISH positive or IHC score $3+$ ), whereas the efficacy was unclear in patients with low HER2 expression (IHC score 0/FISH positive or IHC score 1+/FISH positive). These results were obtained via a subgroup analysis, and may be affected by the smaller number of patients with low HER2 expression than higher HER2 protein expression. Thus, it is premature to conclude that addition of trastuzumab therapy to chemotherapy is not beneficial in patients with low HER 2 expression. Additionally, little has been reported about the clinicopathological features of patients with low HER2 expression [8-10].

In unresectable cases, tumor behavior before treatment is evaluated by biopsy specimens. However, because GC is considered a mixture of heterogeneous tumor types, small biopsy specimens may not reflect its overall behavior, and few studies have focused on HER2-positivity concordance between diagnostic biopsy specimens and surgical specimens $[11,12]$. Because of tumor heterogeneity, the accuracy of HER2 testing can be affected by the site of the examined HER2-stained cells; thus, gastric biopsies could yield false-negative results [13].

We performed a prospective, multicenter, observational cohort study (JFMC44-1101) to evaluate HER2 expression and gene amplification in consecutively registered Japanese patients with metastatic (excluding curatively resected cases) or recurrent GC, and explored the clinicopathological features in relation to HER2 positivity (IHC score 3+ and/or FISH positive) or low HER2 expression (IHC score 0/FISH positive or IHC score 1+/FISH positive). Furthermore, we evaluated the relationship between HER2 protein expression/gene amplification and sampling conditions to ascertain whether HER2 positivity in GC patients can be accurately determined from routinely prepared formalin-fixed, paraffin-embedded tissues.

\section{Methods}

\section{Patients}

JFMC44-1101 is a multicenter, observational cohort study to evaluate HER2 protein expression and gene amplification in consecutively registered Japanese patients with metastatic (excluding curatively resected cases) or recurrent GC. This trial was approved by the central ethics committee of the Japanese Foundation for Multidisciplinary Treatment of Cancer (JFMC) and the institutional review boards of all participating centers. In total, 1427 cases of GC were studied, of which 396 cases were proximal and 1031 were distal. Patients were classified into two groups on the basis of age (younger than 65 years or 65 years or older), according to the WHO classification [14]. All patients provided written informed consent before undergoing study-specific screening procedures. The trial was registered with the University Hospital Medical Information Network (UMIN) Clinical Trials Registry (UMIN ID UMIN000006190). Patient information was collected on the basis of the Japanese Classification of Gastric Carcinoma (third English edition) [15]. 


\section{Selection criteria}

Eligible patients were aged 20 years or older with histologically confirmed adenocarcinoma and in whom metastatic or recurrent GC had been diagnosed after August 2011. Additional eligibility criteria included available pathological tissue samples (six 4- $\mu \mathrm{m}$-thick tissue sections), and written informed patient consent and consent to disseminate the clinical data.

\section{HER2 evaluation}

Excised tissue was formalin fixed and paraffin embedded by conventional histological methods. Six $3-5-\mu \mathrm{m}$ sections were submitted per paraffin-embedded tissue block to allow assessment of the HER2 status: one section was used for each of hematoxylin and eosin staining, IHC, IHC negative control, and FISH, and the remaining two sections were retained as backup specimens. HER2 evaluation was performed centrally with an in vitro diagnosis kit validated by the Japanese Ministry of Health, Labour and Welfare, according to the manufacturer's procedure as follows: tissue sections were tested for HER2 status by IHC with the PATHWAY anti-HER2 (4B5) rabbit monoclonal primary antibody (Roche Diagnostics, Tokyo, Japan), and by FISH with a PathVysion HER-2 DNA probe kit (Abbott Japan, Tokyo, Japan). IHC and FISH results were interpreted centrally, and HER 2 positivity was defined as an IHC score of $3+$ and/or a positive FISH result in accordance with the ToGA study parameters [2]. High HER2 expression was defined as an IHC score of $2+$ /positive FISH result or an IHC score of $3+$, and low HER2 expression was defined as an IHC score of 0/positive FISH result or an IHC score of $1+/$ positive FISH result. The IHC scoring criteria were as follows: IHC score 0 , no staining or membrane staining in less than $10 \%$ of invasive tumor cells; IHC score $1+$, weak membrane staining in $10 \%$ or more of invasive tumor cells; IHC score $2+$, weak to moderate complete or basolateral membrane staining in $10 \%$ or more of invasive tumor cells; and IHC score $3+$, moderate to strong complete or basolateral membrane staining in $10 \%$ or more of invasive tumor cells. To determine FISH-positive status, we determined the fluorescence signal ratio of HER2 (orange) to chromosome enumeration probe 17 (CEP17; green) by counting 20 cancer cells under a fluorescence microscope with a $\times 100$ objective lens. A sample was considered negative for gene amplification (FISH negative) if the HER2-to-CEP17 ratio was less than 2.0, and positive for gene amplification (FISH positive) if the ratio was 2.0 or greater. A HER2-to-CEP17 ratio of 1.8-2.2 (inclusive) was considered equivocal, and was found in 40 cancer cells. Samples were evaluated with a conventional histopathology method, and associations between clinicopathological factors and HER2 positivity or low HER2 expression were examined.

\section{Statistical analysis}

Data were analyzed with the Statistical Package for SAS version 9.2 (SAS Institute, Cary, NC, USA). Fisher's test, Wilcoxon's test, and the chi-squared test were used to test the association between HER2 status and clinicopathological characteristics. To assess the association of HER2 status with clinicopathological features, univariate and multivariate logistic regression analyses were performed. Confidence intervals were computed with the normal approximation of the binomial distribution.

\section{Results}

\section{Patient and sample characteristics}

The trial profile is summarized in Fig. 1. A total of 1461 patients from 157 sites were registered between September 2011 and June 2012. Of these, the HER2 status of 1427 patients was evaluated by both IHC and FISH. Samples were collected from the major tumor site in each patient and were categorized as proximal if they were located in the upper third of the stomach or in the esophagus, and distal if they were situated in the middle third or lower third of the stomach; $27.8 \%$ (396/1427) were proximal GCs and $72.2 \%(1031 / 1427)$ were distal GCs. Patient and sample characteristics at the baseline are summarized in Table 1. The median age of the patients was 68 years (range 23-99 years). The correlations between patient or sample characteristics and HER2 status are summarized in Table 2. Histopathological groupings based on the Lauren classification revealed that 642 patients had intestinal-type tumors and 770 had diffuse-type tumors. Samples were obtained via surgical excision (678 patients) or biopsy (749 patients), and sample collection sites consisted of primary tumors (1348 patients) or metastatic regions (79 patients). HER2-positivity rates in surgically resected tumors and biopsy specimens were significantly different at 18.4 and $23.6 \%$ (Fisher's test, $P=0.016$ ), respectively (Table 2 ). In univariate analysis, the factor biopsy specimen was found to be significantly associated with HER2 positivity (Fig. 2a). However, this association was lost in the multivariate analysis (Fig. 2b).

\section{HER2 positivity and correlation with clinicopathological factors}

The overall HER2-positivity rate (IHC score 3+ and/or FISH positive) was $21.2 \%$ [95\% confidence interval (CI) 
Fig. 1 Trial profile. Human epidermal growth factor receptor 2 (HER2) evaluation by immunohistochemistry and fluorescence in situ hybridization $(F I S H)$ in 1427 samples

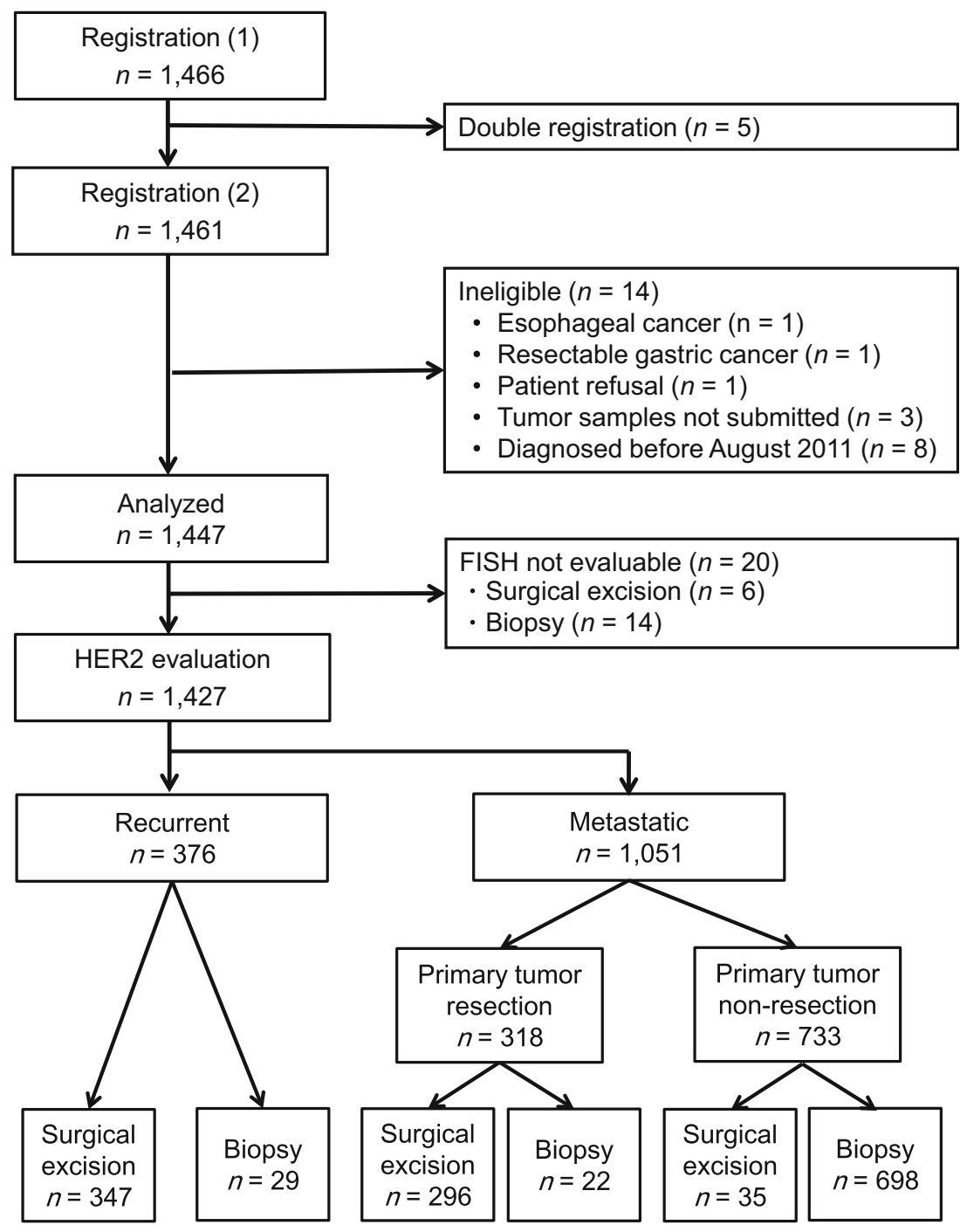

19.1-23.4; 302 of 1427 patients]. There was no significant difference ( $P=0.885$; Fisher's exact test, two-sided) in HER2 positivity between proximal GC cases $(21.5 \% ; 85$ of 396 cases) and distal GC cases $(21.0 \% ; 217$ of 1031 cases). The incidence of high HER2 protein expression (IHC score $3+$ or IHC score $2+$ and FISH positive) was $15.6 \%$ (223 of 1427 patients). FISH was positive in $47.3 \%$ of IHC score $2+$ cases (61 of 129 patients) and $97.5 \%$ of IHC score $3+$ cases (158 of 162 patients) (Table 3 ). In the univariate analysis, HER2 positivity was significantly correlated with sex, histological type, peritoneal metastasis, hepatic metastasis, distant metastasis excluding that detected in the peritoneum, by peritoneal lavage cytology, and in the liver, depth of tumor invasion, macroscopic type, primary tumor location, size, and sample source (Fig. 2a). Multivariate logistic regression analysis revealed that intestinal type, absence of peritoneal metastasis, and hepatic metastasis were independent factors related to HER2 positivity (Fig. 2b). Sampling conditions such as number of biopsy samples, formalin concentration, formalin-fixation time, and sample source had no significant effect on HER2 positivity.

\section{Correlation of HER2 gene amplification by FISH with clinicopathological factors in IHC score $0 / 1+$ cases}

The incidence of low HER2 expression (IHC score 0/FISH positive or IHC score 1+/FISH positive) was $7.0 \%$ (79 of 1136 patients); of these patients, $3.2 \%$ of IHC score 0 cases (19 of 592 patients) and $11 \%$ of IHC score $1+$ cases (60 of 544 patients) were FISH positive (Table 3). In the univariate analysis, low HER 2 expression was significantly correlated with sex, histological type, peritoneal metastasis, hepatic metastasis, depth of tumor invasion, and primary tumor location (Fig. 3a). Finally, multivariate logistic 
Table 1 Characteristics of gastric cancer (GC) patients $(n=1427)$

\begin{tabular}{|c|c|c|c|}
\hline & \multirow{2}{*}{$\begin{array}{l}\text { Recurrent GC } \\
n=376\end{array}$} & \multicolumn{2}{|l|}{ Metastatic GC } \\
\hline & & $\begin{array}{l}\text { Primary tumor resection } \\
n=318\end{array}$ & $\begin{array}{l}\text { Primary tumor no resection } \\
n=733\end{array}$ \\
\hline \multicolumn{4}{|l|}{ Sex } \\
\hline Male & $276(73.4 \%)$ & $215(67.6 \%)$ & $529(72.2)$ \\
\hline Female & $100(26.6 \%)$ & $103(32.4 \%)$ & $204(27.8)$ \\
\hline \multicolumn{4}{|l|}{ Age } \\
\hline Median (years) & 68 & & \\
\hline Range (years) & $23-99$ & & \\
\hline$<65$ years & $158(42.0 \%)$ & $108(34.0 \%)$ & $273(37.2 \%)$ \\
\hline$\geq 65$ years & $218(58.0 \%)$ & $210(66.0 \%)$ & $460(62.8 \%)$ \\
\hline \multicolumn{4}{|l|}{ PS (ECOG) } \\
\hline 0 & $242(64.4 \%)$ & $180(56.6 \%)$ & $438(59.8 \%)$ \\
\hline $1,2,3,4$ & $134(35.6 \%)$ & $138(43.4 \%)$ & $295(40.2 \%)$ \\
\hline \multicolumn{4}{|l|}{ Source of sample } \\
\hline Biopsy & $29(7.7 \%)$ & $22(6.9 \%)$ & $698(95.2 \%)$ \\
\hline Surgical excision & $347(92.3 \%)$ & $296(93.1 \%)$ & $35(4.8 \%)$ \\
\hline \multicolumn{4}{|c|}{ Depth of tumor invasion } \\
\hline T1a & $4(1.1 \%)$ & 0 & $1(0.1 \%)$ \\
\hline $\mathrm{T} 1 \mathrm{~b}$ & $19(5.1 \%)$ & $4(1.3 \%)$ & $4(0.5 \%)$ \\
\hline $\mathrm{T} 2$ & $45(12.0 \%)$ & $11(3.5 \%)$ & $20(2.7 \%)$ \\
\hline T3 & $121(32.2 \%)$ & $36(11.3 \%)$ & $142(19.4 \%)$ \\
\hline $\mathrm{T} 4 \mathrm{a}$ & $153(40.7 \%)$ & $200(62.9 \%)$ & $367(50.1 \%)$ \\
\hline $\mathrm{T} 4 \mathrm{~b}$ & $33(8.8 \%)$ & $63(19.8 \%)$ & $160(21.8 \%)$ \\
\hline $\mathrm{Tx}$ & 0 & $4(1.3 \%)$ & $39(5.3 \%)$ \\
\hline Unclear & $1(0.3 \%)$ & & \\
\hline \multicolumn{4}{|c|}{ Lymph node metastasis } \\
\hline No & $65(17.3 \%)$ & $22(6.9 \%)$ & $93(12.7 \%)$ \\
\hline N1 & $57(15.2 \%)$ & $39(12.3 \%)$ & $44(6.0 \%)$ \\
\hline $\mathrm{N} 2$ & $76(20.2 \%)$ & $43(13.5 \%)$ & $108(14.7 \%)$ \\
\hline N3a & $106(28.2 \%)$ & $75(23.6 \%)$ & $83(11.3 \%)$ \\
\hline $\mathrm{N} 3 \mathrm{~b}$ & $61(16.2 \%)$ & $102(32.1 \%)$ & $25(3.4 \%)$ \\
\hline NX & $11(2.9 \%)$ & $37(11.6 \%)$ & $380(51.8 \%)$ \\
\hline \multicolumn{4}{|c|}{ Peritoneal metastasis } \\
\hline $\mathrm{P} 0$ & $358(95.2 \%)$ & $159(50.0 \%)$ & $192(26.2 \%)$ \\
\hline $\mathrm{P} 1$ & $10(2.7 \%)$ & $151(47.5 \%)$ & $333(45.4 \%)$ \\
\hline Unclear & $8(2.1 \%)$ & $8(2.5)$ & $208(28.4 \%)$ \\
\hline \multicolumn{4}{|c|}{ Peritoneal lavage cytology } \\
\hline CY0 & $316(84.0 \%)$ & $115(36.2 \%)$ & $85(11.6 \%)$ \\
\hline CY1 & 0 & $159(50.0 \%)$ & $161(22.0 \%)$ \\
\hline Unclear & $60(16.0 \%)$ & $44(13.8 \%)$ & $487(66.4 \%)$ \\
\hline \multicolumn{4}{|l|}{ Hepatic metastasis } \\
\hline HO & $371(98.7 \%)$ & $257(80.8 \%)$ & $493(67.3 \%)$ \\
\hline $\mathrm{H} 1$ & $3(0.8 \%)$ & $57(17.9 \%)$ & $209(28.5 \%)$ \\
\hline Unclear & $2(0.5 \%)$ & $4(1.3 \%)$ & $31(4.2 \%)$ \\
\hline
\end{tabular}

ECOG Eastern Cooperative Oncology Group, PS performance status regression analysis revealed that age (65 years or older), intestinal type, and T1-T3 stage were independent factors related to low HER2 expression (Fig. 3b). We performed ad hoc analysis in the surgical specimen group. In the univariate analysis $(n=569)$, low HER 2 expression was significantly correlated with sex (odds ratio $0.409,95 \%$ CI 
Table 2 Correlation between patient and sample characteristics and human epidermal growth factor receptor $2(H E R 2)$ status $(n=1427)$

\begin{tabular}{|c|c|c|c|c|}
\hline & Number & HER2 positivity (\%) & HER2 positive $(n=302)$ & HER2 negative $(n=1125)$ \\
\hline \multicolumn{5}{|l|}{ Diagnosis status } \\
\hline Metastatic & 1051 & 22.2 & 233 & 818 \\
\hline Recurrent & 376 & 18.4 & 69 & 307 \\
\hline \multicolumn{5}{|c|}{ Time to recurrence } \\
\hline$<18$ months & 212 & 20.3 & 43 & 169 \\
\hline$\geq 18$ months & 164 & 15.9 & 26 & 138 \\
\hline \multicolumn{5}{|l|}{ Sex } \\
\hline Male & 1020 & 23.7 & 242 & 778 \\
\hline Female & 407 & 14.7 & 60 & 347 \\
\hline \multicolumn{5}{|l|}{ Age } \\
\hline$<65$ years & 539 & 18.7 & 101 & 438 \\
\hline$\geq 65$ years & 888 & 22.6 & 201 & 687 \\
\hline \multicolumn{5}{|c|}{ Tumor location: three gastric regions (major site) } \\
\hline $\mathrm{U}$ & 391 & 21.2 & 83 & 308 \\
\hline M & 548 & 19.9 & 109 & 439 \\
\hline $\mathrm{L}$ & 480 & 22.3 & 107 & 373 \\
\hline Other $(\mathrm{E}$ or $\mathrm{D})$ & 6 & 33.3 & 2 & 4 \\
\hline \multicolumn{5}{|c|}{ Tumor location: cross-sectional part (major site) } \\
\hline Less & 550 & 22.0 & 121 & 429 \\
\hline Gre & 202 & 23.8 & 48 & 154 \\
\hline Ant & 142 & 21.8 & 31 & 111 \\
\hline Post & 188 & 20.2 & 38 & 150 \\
\hline Circ & 332 & 18.1 & 60 & 272 \\
\hline \multicolumn{5}{|l|}{ Macroscopic type } \\
\hline Type 0 & 46 & 28.3 & 13 & 33 \\
\hline Type 1 & 40 & 30.0 & 12 & 28 \\
\hline Type 2 & 291 & 26.5 & 77 & 214 \\
\hline Type 3 & 639 & 23.6 & 151 & 488 \\
\hline Type 4 & 353 & 11.3 & 40 & 313 \\
\hline Type 5 & 55 & 14.5 & 8 & 47 \\
\hline \multicolumn{5}{|c|}{ Histological classification $^{\mathrm{a}}$} \\
\hline pap & 38 & 36.8 & 14 & 24 \\
\hline tub1 & 155 & 38.1 & 59 & 96 \\
\hline tub2 & 353 & 33.1 & 117 & 236 \\
\hline por1 & 359 & 19.8 & 71 & 288 \\
\hline por2 & 347 & 7.8 & 27 & 320 \\
\hline sig & 134 & 6.7 & 9 & 125 \\
\hline muc & 41 & 12.2 & 5 & 36 \\
\hline \multicolumn{5}{|c|}{ Lauren classification $^{\mathrm{b}}$} \\
\hline Intestinal & 642 & 32.7 & 210 & 432 \\
\hline Diffuse & 770 & 11.7 & 90 & 680 \\
\hline \multicolumn{5}{|c|}{ Peritoneal metastasis } \\
\hline P0 & 709 & 23.1 & 164 & 545 \\
\hline $\mathrm{P} 1$ & 494 & 14.2 & 70 & 424 \\
\hline \multicolumn{5}{|c|}{ Peritoneal lavage cytology } \\
\hline CY0 & 516 & 18.6 & 96 & 420 \\
\hline CY1 & 320 & 15.9 & 51 & 269 \\
\hline
\end{tabular}


Table 2 continued

\begin{tabular}{|c|c|c|c|c|}
\hline & Number & HER2 positivity (\%) & HER2 positive $(n=302)$ & HER2 negative $(n=1125)$ \\
\hline \multicolumn{5}{|l|}{ Hepatic metastasis } \\
\hline $\mathrm{HO}$ & 1121 & 18.0 & 202 & 919 \\
\hline H1 & 269 & 34.9 & 94 & 175 \\
\hline \multicolumn{5}{|l|}{ Distant metastasis $^{\mathrm{c}}$} \\
\hline $\mathrm{dM} 0$ & 934 & 18.0 & 168 & 766 \\
\hline $\mathrm{dM} 1$ & 446 & 28.0 & 125 & 321 \\
\hline \multicolumn{5}{|c|}{ Lymph node metastasis } \\
\hline No & 180 & 12.8 & 23 & 157 \\
\hline N1 & 140 & 23.6 & 33 & 107 \\
\hline $\mathrm{N} 2$ & 227 & 21.6 & 49 & 178 \\
\hline N3a & 264 & 24.6 & 65 & 199 \\
\hline $\mathrm{N} 3 \mathrm{~b}$ & 188 & 13.8 & 26 & 162 \\
\hline \multicolumn{5}{|c|}{ Depth of tumor invasion } \\
\hline $\mathrm{T} 1$ & 32 & 37.5 & 12 & 20 \\
\hline $\mathrm{T} 2$ & 76 & 25.0 & 19 & 57 \\
\hline $\mathrm{T} 3$ & 299 & 28.4 & 85 & 214 \\
\hline $\mathrm{T} 4 \mathrm{a}$ & 720 & 16.5 & 119 & 601 \\
\hline $\mathrm{T} 4 \mathrm{~b}$ & 256 & 21.5 & 55 & 201 \\
\hline \multicolumn{5}{|l|}{ Source of sample } \\
\hline Surgical excision & 678 & 18.4 & 125 & 553 \\
\hline Biopsy & 749 & 23.6 & 177 & 572 \\
\hline \multicolumn{5}{|c|}{ No. of biopsy samples } \\
\hline $1-3$ & 339 & 23.0 & 78 & 261 \\
\hline $4-8$ & 378 & 24.1 & 91 & 287 \\
\hline$\geq 9$ & 31 & 25.8 & 8 & 23 \\
\hline \multicolumn{5}{|c|}{ Formalin concentration } \\
\hline $10 \%$ & 950 & 20.7 & 197 & 753 \\
\hline $15 \%$ & 129 & 16.3 & 21 & 108 \\
\hline $20 \%$ & 335 & 25.1 & 84 & 251 \\
\hline$>20 \%$ & 6 & 0.0 & 0 & 6 \\
\hline \multicolumn{5}{|c|}{ Formalin fixation time } \\
\hline$<18 \mathrm{~h}$ & 497 & 22.9 & 114 & 383 \\
\hline$\geq 18 \mathrm{~h},<24 \mathrm{~h}$ & 462 & 21.2 & 98 & 364 \\
\hline$\geq 24 \mathrm{~h},<48 \mathrm{~h}$ & 269 & 17.5 & 47 & 222 \\
\hline$\geq 48 \mathrm{~h}$ & 186 & 22.6 & 42 & 144 \\
\hline \multicolumn{5}{|c|}{ Sample collection sites } \\
\hline Primary tumor & 1348 & 21.7 & 292 & 1056 \\
\hline Metastatic region & 79 & 12.7 & 10 & 69 \\
\hline
\end{tabular}

Ant anterior wall, Circ circumferential, $D$ duodenum, $E$ esophagus, Gre greater curvature, $L$ lower third, Less lesser curvature, $M$ middle third, тис mucinous adenocarcinoma, pap papillary adenocarcinoma, por1 solid-type poorly differentiated adenocarcinoma, por 2 non-solid-type poorly differentiated adenocarcinoma, Post posterior wall, $U$ upper third, sig signet ring cell carcinoma, tubl well-differentiated tubular adenocarcinoma, tub2 moderately differentiated tubular adenocarcinoma

${ }^{a}$ Histological features were classified on the basis of the Japanese Classification of Gastric Carcinoma (third English edition)

${ }^{b}$ For Lauren classification, pap, tub, and por1 of type 1 or type 2 were defined as intestinal type, and the others were defined as diffuse type

${ }^{c}$ Distant metastasis was defined as metastasis to other organs excluding that detected in the peritoneum, by peritoneal lavage cytology, and in the liver

0.178-0.940, $P=0.035$ ), histological type (odds ratio $0.257,95 \%$ CI $0.131-0.507, P<0.001)$, hepatic metastasis (odds ratio 4.598, $95 \%$ CI 2.013-10.505, $P<0.001$ ), depth of tumor invasion (odds ratio 0.405, $95 \%$ CI $0.215-0.763, P=0.005)$, and formalin concentration (odds ratio $1.949,95 \%$ CI $1.035-3.669, P=0.039$ ). 

epidermal growth factor receptor 2 (HER2) positivity a Univariate analysis of HER2 positivity

(immunohistochemistry score

$3+$ and/or fluorescence in situ hybridization positive) in samples from gastric cancer (GC) patients. b Multivariate analysis of HER2-positivity in samples from GC patients $(n=1088)$. Red squares indicate a significant association with HER2 status (HER2 are two-sided, with $P<0.05$ indicating statistical significance. $C I$ confidence interval, $C Y$ peritoneal lavage cytology, $d M$ distant metastasis excluding that detected in the peritoneum, by peritoneal lavage cytology, and in the liver, $H$ hepatic metastasis, $N$ lymph node metastasis, $P$ peritoneal metastasis, $P S$ performance status, $T$ depth of tumor invasion (color figure online)
Fig. 2 Correlation of human with clinicopathological factors. positive/negative). All $P$ values

\begin{tabular}{|c|c|c|c|c|c|c|}
\hline & $\mathrm{n}$ & & & Odds ratio & $95 \% \mathrm{Cl}$ & $P$ \\
\hline $\begin{array}{l}\text { Metastatic } \\
\text { vs. recurrence }\end{array}$ & 1427 & & & 0.79 & $0.59-1.06$ & 0.120 \\
\hline $\begin{array}{l}\text { Time to recurrence } \\
\text { (<18 vs. } \geq 18 \text { years) }\end{array}$ & 376 & & & 0.74 & $0.43-1.27$ & 0.272 \\
\hline $\begin{array}{l}\text { Sex } \\
\text { (male vs. female) }\end{array}$ & 1427 & $-\square$ & & 0.56 & $0.41-0.76$ & $<0.001$ \\
\hline Age (<65 vs. $\geq 65$ years) & 1427 & & & 1.27 & $0.97-1.66$ & 0.081 \\
\hline $\begin{array}{l}\text { Lauren classification } \\
\text { (intestinal vs. diffuse) }\end{array}$ & 1412 & $\rightarrow-$ & & 0.27 & $0.21-0.36$ & $<0.001$ \\
\hline P0 vs. P1 & 1203 & $\neg-$ & & 0.55 & $0.40-0.75$ & $<0.001$ \\
\hline CY0 vs. CY1 & 836 & & & 0.83 & $0.57-1.20$ & 0.325 \\
\hline $\mathrm{H} 0$ vs. $\mathrm{H} 1$ & 1390 & & $-\square-$ & 2.44 & $1.82-3.28$ & $<0.001$ \\
\hline dM0 vs. dM1 & 1380 & & $-\square$ & 1.78 & $1.36-2.32$ & $<0.001$ \\
\hline N0, N1, N2 vs. N3 & 999 & & & 1.06 & $0.78-1.45$ & 0.710 \\
\hline T1, T2, T3 vs. T4 & 1383 & $-\square$ & & 0.54 & $0.42-0.71$ & $<0.001$ \\
\hline $\begin{array}{l}\text { Macroscopic type } \\
(1,2 \text { vs. } 0,3,4)\end{array}$ & 1369 & & & 0.67 & $0.50-0.89$ & 0.005 \\
\hline $\begin{array}{l}\text { Three gastric regions } \\
\text { (single vs. multiple) }\end{array}$ & 1427 & & & 0.74 & $0.58-0.96$ & 0.023 \\
\hline $\begin{array}{l}\text { Cross-sectional parts } \\
\text { (single vs. multiple) }\end{array}$ & 1427 & & & 0.99 & $0.76-1.28$ & 0.925 \\
\hline $\begin{array}{l}\text { Greatest dimension } \\
(<6 \mathrm{~cm} \text { vs. } \geq 6 \mathrm{~cm})\end{array}$ & 991 & & & 0.73 & $0.53-1.00$ & 0.048 \\
\hline PS (0 vs. $1,2,3,4)$ & 1427 & & & 0.87 & $0.67-1.13$ & 0.290 \\
\hline $\begin{array}{l}\text { Biopsy vs. } \\
\text { surgical excision }\end{array}$ & 1427 & $-\square-$ & & 0.73 & $0.57-0.95$ & 0.017 \\
\hline $\begin{array}{l}\text { No. of biopsy samples } \\
(\leq 3 \text { vs. } \geq 4)\end{array}$ & 748 & & & 1.07 & $0.76-1.50$ & 0.702 \\
\hline $\begin{array}{l}\text { Formalin concentration } \\
(10 \% \text { vs. }>10 \%)\end{array}$ & 1420 & & & 1.10 & $0.84-1.44$ & 0.487 \\
\hline $\begin{array}{l}\text { Formalin-fixation time } \\
(<24 \text { h vs. } \geq 24 \text { h) }\end{array}$ & 1414 & & & 0.86 & $0.65-1.13$ & 0.275 \\
\hline $\begin{array}{l}\text { Sample collection site } \\
\text { (primary vs. metastasis) }\end{array}$ & 1427 & & & 0.52 & $0.27-1.03$ & 0.061 \\
\hline
\end{tabular}

0.2

5

However, multivariate logistic regression analysis revealed that there were no independent factors related to low HER2 expression in the 392 of 569 patients for whom data were available.

\section{Discussion}

Previous studies reported that the rate of HER2 positivity (IHC score $3+$ and/or FISH positive) in Japanese GC patients was approximately 10-20\% [5-7], but testing methods and interpretation criteria were not standardized. In this study, HER2 status was centrally assessed with a standardized method, which was used to prospectively interpret both the IHC data and the FISH data of the ToGA study; The rate of HER 2 positivity was $21.2 \%$ in Japanese patients, identical to the ToGA screening population [3]. The rate of HER 2 positivity was reported as $27 \%$ in Japanese patients in the ToGA study [4], higher than previously reported rates. This might be a result of bias toward patient selection from past reports [16-18], because the primary purpose of the ToGA study was to assess the clinical efficacy and safety of trastuzumab rather than to evaluate HER2 positivity. The incidence of higher HER2 protein expression (IHC score $2+$ /FISH positive or IHC score $3+; 15.6 \%$ ) and the proportions of FISH positivity 
Fig. 2 continued

\begin{tabular}{|c|c|c|c|c|c|}
\hline & & & Jdds ratio & $95 \% \mathrm{Cl}$ & $P$ \\
\hline $\begin{array}{l}\text { Metastatic } \\
\text { vs. recurrence }\end{array}$ & $\longrightarrow$ & - & 0.80 & $0.49-1.31$ & 0.380 \\
\hline $\begin{array}{l}\text { Sex } \\
\text { (male vs. female) }\end{array}$ & & & 0.72 & $0.48-1.07$ & 0.102 \\
\hline Age (<65 vs. $\geq 65$ years) & & $1-$ & 1.19 & $0.85-1.68$ & 0.317 \\
\hline $\begin{array}{l}\text { Lauren classification } \\
\text { (intestinal vs. diffuse) }\end{array}$ & $\square-$ & & 0.28 & $0.19-0.41$ & $<0.001$ \\
\hline P0 vs. P1 & $\square-$ & & 0.58 & $0.39-0.86$ & 0.007 \\
\hline H0 vs. H1 & & $\square$ & 1.61 & $1.05-2.48$ & 0.029 \\
\hline dM0 vs. dM1 & & - & 1.45 & $0.98-2.14$ & 0.061 \\
\hline T1, T2, T3 vs. T4 & $\rightarrow$ & & 0.76 & $0.53-1.10$ & 0.144 \\
\hline $\begin{array}{l}\text { Macroscopic type } \\
(1,2 \text { vs. } 0,3,4)\end{array}$ & & & 1.33 & $0.91-1.94$ & 0.145 \\
\hline $\begin{array}{l}\text { Three gastric regions } \\
\text { (single vs. multiple) }\end{array}$ & & & 0.86 & $0.60-1.22$ & 0.395 \\
\hline $\begin{array}{l}\text { Cross-sectional parts } \\
\text { (single vs. multiple) }\end{array}$ & & & 1.40 & $0.99-1.98$ & 0.054 \\
\hline PS (0 vs. $1,2,3,4)$ & $\rightarrow$ & & 0.80 & $0.57-1.11$ & 0.178 \\
\hline $\begin{array}{l}\text { Biopsy } \\
\text { vs. surgical excision }\end{array}$ & & & 0.87 & $0.59-1.28$ & 0.482 \\
\hline $\begin{array}{l}\text { Formalin concentration } \\
(10 \% \text { vs. }>10 \%)\end{array}$ & & & 1.19 & $0.86-1.66$ & 0.300 \\
\hline $\begin{array}{l}\text { Formalin-fixation time } \\
(<24 \text { h vs. } \geq 24 \text { h) }\end{array}$ & & & 0.95 & $0.67-1.36$ & 0.785 \\
\hline $\begin{array}{l}\text { Sample collection site } \\
\text { (primary vs. metastasis) }\end{array}$ & & - & 0.69 & $0.32-1.50$ & 0.350 \\
\hline
\end{tabular}

Table 3 Human epidermal growth factor receptor 2 positive rates as assessed by fluorescence in situ hybridization $(F I S H)$ and immunohistochemistry $(I H C)$

\begin{tabular}{|c|c|c|c|c|c|}
\hline \multirow[t]{2}{*}{ FISH result } & \multicolumn{4}{|l|}{ IHC score } & \multirow[t]{2}{*}{ Total } \\
\hline & 0 & $1+$ & $2+$ & $3+$ & \\
\hline Negative & $573(96.8 \%)$ & $484(89.0 \%)$ & $68(52.7 \%)$ & $4(2.5 \%)$ & $1129(79.1 \%)$ \\
\hline Positive & $19(3.2 \%)$ & $60(11.0 \%)$ & $61(47.3 \%)$ & $158(97.5 \%)$ & $298(20.9 \%)$ \\
\hline Total & $592(100 \%)$ & $544(100 \%)$ & $129(100 \%)$ & $162(100 \%)$ & $1427(100 \%)$ \\
\hline
\end{tabular}

in IHC score 0 and IHC score $1+$ cases (3.2 and $11 \%$ ) were comparable with those reported in the ToGA study [3]. Similarly, the concordance between IHC and FISH in our results is consistent with that reported in the ToGA study.

A high correlation between HER2 positivity and histological subtype was reported by several authors [19-24]. In the ToGA study, HER2 positivity varied significantly according to histological subtype (intestinal type $31.8 \%$; diffuse type $6.1 \%$; mixed type $20 \%$ ) [3]; thus, intestinal type was strongly correlated with HER2 expression. Several reports indicated that intestinal type is associated with hematogenous metastasis, particularly to the liver [25], and with older age [26], whereas the diffuse type is adversely related to peritoneal dissemination [27]. In the present study, intestinal type, absence of peritoneal metastasis, and hepatic metastasis were shown to be independent factors related to HER2 positivity in a multivariate logistic regression analysis. This agrees with what is known about the histological type, i.e., intestinal or diffuse, and the 
Fig. 3 Correlation of human epidermal growth factor receptor 2 (HER2) gene amplification assessed by fluorescence in situ hybridization (FISH) with clinicopathological factors in immunohistochemistry (IHC) score $0 / 1+$ cases. a Univariate analysis of low HER2 expression as assessed by IHC score 0/FISH-positive or IHC score $1+/$ FISH-positive samples from gastric cancer patients. b Multivariate analysis of low HER 2 expression as assessed by IHC score 0/FISH-positive or IHC score 1+/FISH-positive samples from gastric cancer patients $(n=874)$. Red squares indicate a significant association with HER2 status (IHC score 0/FISH positive or IHC score 1+/FISH positive). All $P$ values are two-sided, with $P<0.05$ indicating statistical significance. $C I$ confidence interval, $C Y$ peritoneal lavage cytology, $d M$ distant metastasis excluding that detected in the peritoneum, by peritoneal lavage cytology, and in the liver, $H$ hepatic metastasis, $N$ lymph node metastasis, $P$ peritoneal metastasis, $P S$ performance status, $T$ depth of tumor invasion (color figure online)

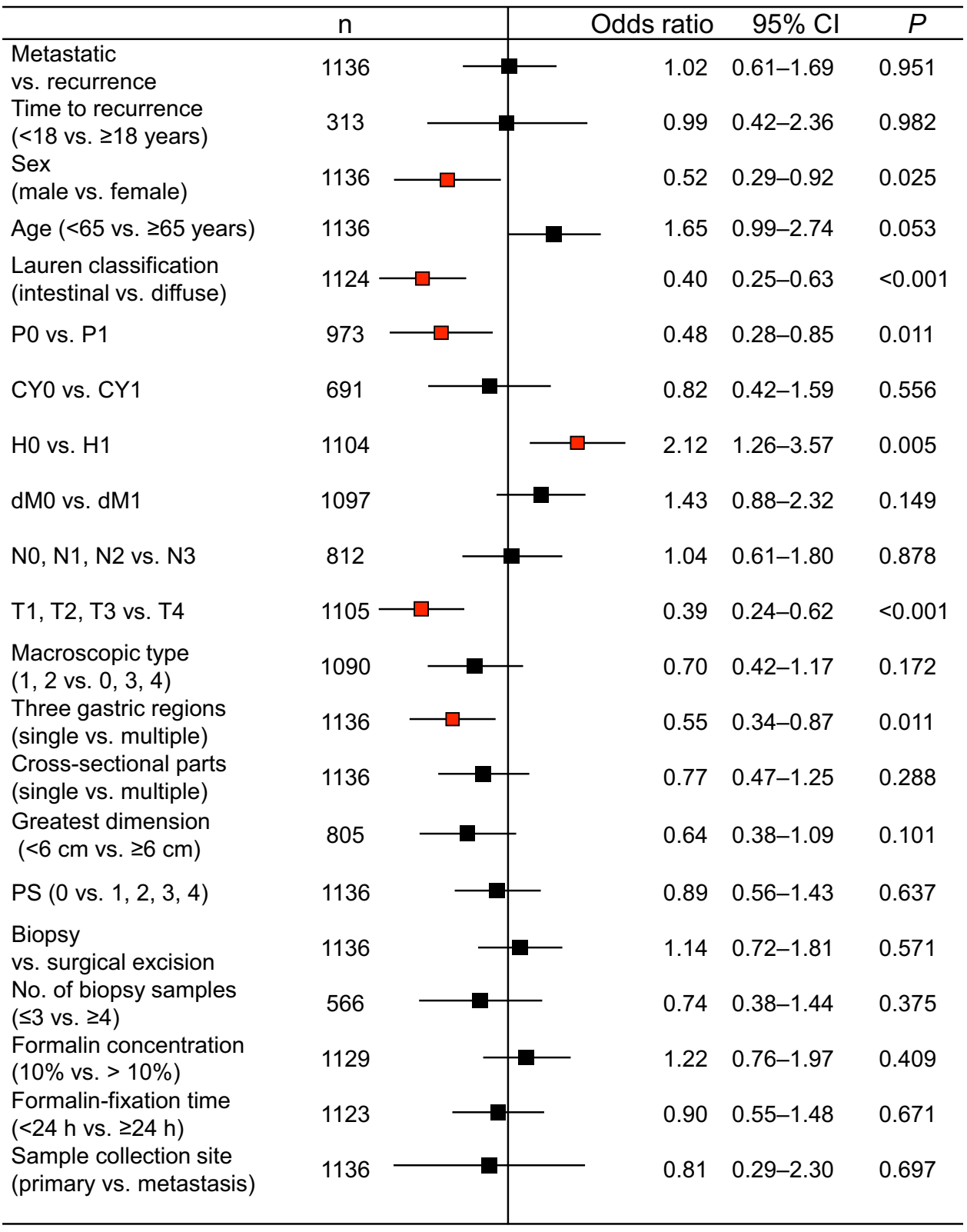

association with accompanying hepatic or peritoneal metastasis, respectively.

Moreover, intestinal type, age (65 years or older), and T1-T3 stage were independent factors related to low HER2 expression (IHC score $0 / 1+$ and FISH positive). This result reveals that HER2-related factors are associated with intestinal-type GCs. Diffuse-type GCs are more malignant than their intestinal-type counterparts, demonstrating early invasion into the muscularis propria [25]. A previous report demonstrated that diffuse-type advanced GC was significantly associated with advanced pathological T stage [28]. Thus, diffuse type is commoner in T4 tumors, whereas intestinal type is commoner in T1-T3 tumors. As intestinal type is the most robust factor related to HER2 expression, T1-T3 stage may be an independent factor related to low HER2 expression even in intestinal-type IHC score $0 / 1+$ GC cases. However, the current study was limited by the extent and accuracy of the $\mathrm{T}$ staging, which was determined by either pathological or clinical diagnosis methods. To resolve these limitations, we performed ad hoc analyses for low HER2 expression (IHC score 0/FISH positive or IHC score $1+/$ FISH positive) in the surgical specimen group, because the $\mathrm{T}$ stage in the surgical samples was accurately determined pathologically. T1-T3 stage was 
Fig. 3 continued

\begin{tabular}{|c|c|c|c|}
\hline & Odds ratio & $95 \% \mathrm{Cl}$ & $P$ \\
\hline $\begin{array}{l}\text { Metastatic } \\
\text { vs. recurrence }\end{array}$ & 0.57 & $0.25-1.34$ & 0.201 \\
\hline $\begin{array}{l}\text { Sex } \\
\text { (male vs. female) }\end{array}$ & 0.54 & $0.26-1.10$ & 0.091 \\
\hline Age (<65 vs. $\geq 65$ years) & 2.10 & $1.10-4.00$ & 0.024 \\
\hline $\begin{array}{l}\text { Lauren classification } \\
\text { (intestinal vs. diffuse) }\end{array}$ & 0.50 & $0.26-0.94$ & 0.030 \\
\hline P0 vs. P1 & 0.51 & $0.25-1.05$ & 0.068 \\
\hline $\mathrm{H} 0$ vs. $\mathrm{H} 1$ & 1.19 & $0.54-2.61$ & 0.662 \\
\hline dM0 vs. dM1 & 1.10 & $0.54-2.24$ & 0.788 \\
\hline T1, T2, T3 vs. T4 & 0.48 & $0.26-0.89$ & 0.020 \\
\hline $\begin{array}{l}\text { Macroscopic type } \\
(1,2 \text { vs. } 0,3,4)\end{array}$ & 1.39 & $0.71-2.70$ & 0.337 \\
\hline $\begin{array}{l}\text { Three gastric regions } \\
\text { (single vs. multiple) }\end{array}$ & 0.73 & $0.39-1.35$ & 0.312 \\
\hline $\begin{array}{l}\text { Cross-sectional parts } \\
\text { (single vs. multiple) }\end{array}$ & 1.06 & $0.57-1.98$ & 0.847 \\
\hline PS (0 vs. $1,2,3,4)$ & 0.72 & $0.40-1.28$ & 0.258 \\
\hline $\begin{array}{l}\text { Biopsy } \\
\text { vs. surgical excision }\end{array}$ & 1.33 & $0.69-2.58$ & 0.395 \\
\hline $\begin{array}{l}\text { Formalin concentration } \\
(10 \% \text { vs. }>10 \%)\end{array}$ & 1.27 & $0.72-2.24$ & 0.408 \\
\hline $\begin{array}{l}\text { Formalin-fixation time } \\
(<24 \text { h vs. } \geq 24 \text { h })\end{array}$ & 0.71 & $0.38-1.33$ & 0.282 \\
\hline $\begin{array}{l}\text { Sample collection site } \\
\text { (primary vs. metastasis) }\end{array}$ & 0.87 & $0.25-3.04$ & 0.833 \\
\hline
\end{tabular}

statistically significantly correlated with low HER2 expression in the univariate analysis, but was not significantly correlated in the multivariate analysis. Likewise, intestinal type, sex, hepatic metastasis, and formalin concentration were statistically significantly associated with low HER2 expression in the univariate analysis; however, there were no significant differences in the multivariate analysis. The discrepancies in these analyses may result from the multivariate analysis being performed only in 392 of 569 cases owing to missing data in the remaining cases, thereby conferring a lack of statistical significance. Further studies are required to confirm this result, and considering these limitations, we cannot conclude that depth of tumor invasion is a factor related to low HER2 expression.

There are several factors that are reported to affect HER2 staining results, such as type of fixative, total fixation time, fixative $\mathrm{pH}$, tissue type, and time before fixation. In the present study, we evaluated the relationship between
HER2 expression and sampling conditions; however, the number of biopsy samples, formalin concentration, and formalin-fixation time had no significant effect on HER2 positivity and low HER2 expression. Unfortunately, the recommended conditions for fixation could not be adhered to in this study because the biopsy specimens and surgically resected specimens were mixed up and because correlations between formalin concentration and fixation time could not be undertaken. Moreover, the time before fixation (so-called cold ischemia) and the specimen size were unclear. Further prospective studies aiming to comprehensively evaluate the effects of formalin concentration, formalin-fixation time, and cold ischemia on HER2 testing are required.

There was concern that examination of gastric biopsy samples alone might introduce false-positive and/or falsenegative data, because HER2 intratumoral heterogeneity in GC is observed in 20-70\% of HER2-positive tumors [13, 
29] and is the major cause of discrepancies between biopsy samples and surgical specimens. In the multivariate analysis of the present study results, HER2-positivity rates in surgically resected tumors and biopsy samples were not significantly different, similar to the findings in the HEREAGLE study [24]. However, these studies were limited in that the correlation between surgical specimens and biopsy samples was not paired, although this contrasts with the GERCOR study, where the overall concordance rate between surgical specimens and paired biopsy samples reached $94 \%$ [12]. We also examined the concordance between predominant histological type and histological type with a HER2-positive component, which was determined as $81.3 \%$ with the Lauren classification (data not shown). Approximately $20 \%$ of cases showed a discrepancy; therefore, gastroenterologists should consider performing multiple biopsy sampling from varied collection sites to overcome tumor heterogeneity in GC.

In conclusion, HER2 expression in a Japanese GC population was similar in distribution to that identified in the ToGA study. Intestinal type was revealed as an independent factor related to both HER2 positivity and low HER2 expression.

Acknowledgments This trial was supported by the Japanese Foundation for Multidisciplinary Treatment of Cancer. Editorial support was provided by H. Nikki March and was funded by Chugai Pharmaceutical Co. Ltd.

Conflict of interest S. Matsusaka has received commercial research grants from Taiho Pharmaceutical Co. Ltd. K. Nishikawa has received commercial research grants and personal fees from Yakult Honsha Co. Ltd, Taiho Pharmaceutical Co. Ltd, and Ajinomoto Pharmaceuticals, and personal fees from Chugai Pharmaceutical Co. Ltd. Tsumura Co. Ltd, and Terumo Corporation. T. Yoshikawa received commercial research grants and personal fees from Chugai Pharmaceutical Co. Ltd during the conduct of the study, has received grants and personal fees from Taiho Pharmaceutical Co. Ltd, Novartis Pharma K.K., Ono Pharmaceutical Co. Ltd, Yakult Honsha Co. Ltd, Covidien Japan Co. Ltd, and the nonprofit organization Kanagawa Standard Anti-cancer Therapy Support System, has received personal fees from Eli Lilly Japan K.K., Abbott Japan Co. Ltd, Kaken Pharmaceutical Co. Ltd, Johnson \& Johnson K.K., Takeda Pharmaceutical Co. Ltd, Secom Medical System Co. Ltd, Nikkei Business Publications, and the nonprofit organizations Tokyo Cooperative Oncology Group, Clinical Training Support Council, and Cancer Net Japan, and has received grants from Daiichi Sankyo Co. Ltd and Nippon Kayaku Co. Ltd. A. Ochiai has received commercial research grants from Bayer Ltd, Ventana Medical Systems Inc., Eli Lilly Japan K.K., Toray Industries Inc., Takeda Pharmaceutical Co. Ltd, Taiho Pharmaceutical Co. Ltd, Merck Serono Co. Ltd, Janssen Pharmaceutical K.K., Eisai Co. Ltd, Otsuka Pharmaceutical Co. Ltd, and Daiichi Sankyo Co. Ltd, and nonfinancial support from Fujifilm Co. Ltd. S. Morita has received commercial research grants and personal fees from Chugai Pharmaceutical Co. Ltd. T. Sano has received personal fees from Taiho Pharmaceutical Co. Ltd, Sanofi K.K., Yakult Honsha Co. Ltd, and Chugai Pharmaceutical Co. Ltd. Y. Kodera has received commercial research grants and personal fees from Chugai Pharmaceutical Co. Ltd, grants and personal fees from Taiho Pharmaceutical Co. Ltd, Sanofi K.K.,
Bristol-Myers Squib Ltd, Merck Serono Co. Ltd, Yakult Honsha Co. Ltd, Daiichi Sankyo Co. Ltd, Otsuka Pharmaceutical Co. Ltd, Takeda Pharmaceutical Co. Ltd, Johnson \& Johnson K.K., Asahi Kasei Pharma Corporation, Shionogi \& Co. Ltd, Eli Lilly Japan K.K., Pfizer Japan Inc., Ajinomoto Pharmaceuticals, Ono Pharmaceutical Co. Ltd, Kaken Pharmaceutical Co. Ltd, Covidien Japan Co. Ltd, Tsumura Co. Ltd, and grants from Eisai Co. Ltd, Abbott Japan Co. Ltd, CSL Behring Ltd, Teijin Pharma Ltd, the Japan Blood Products Organization, Torii Pharmaceutical Co. Ltd, and Mitsubishi Tanabe Pharma Corporation. Y. Kakeji has received personal fees from Chugai Pharmaceutical Co. Ltd. J. Sakamoto has received personal fees from Takeda Pharmaceutical Co. Ltd and Tsumura Co. Ltd. K. Yoshida received commercial research grants from Chugai Pharmaceutical Co. Ltd during the conduct of the study, has received grants and personal fees from Taiho Pharmaceutical Co. Ltd, Pfizer Japan Inc., Chugai Pharmaceutical Co. Ltd, Yakult Honsha Co. Ltd, Bristol-Myers Squibb Ltd, and Kyowa Hakko Kirin Co. Ltd, honoraria from Taiho Pharmaceutical Co. Ltd, Pfizer Japan Inc., Chugai Pharmaceutical Co. Ltd, Kyowa Hakko Kirin Co. Ltd, and Yakult Honsha Co. Ltd, and has acted as a consultant or advisor for Taiho Pharmaceutical Co. Ltd and F. Hoffmann-La Roche Ltd. All remaining authors declare that they have no conflict of interest.

Open Access This article is distributed under the terms of the Creative Commons Attribution 4.0 International License (http://crea tivecommons.org/licenses/by/4.0/), which permits unrestricted use, distribution, and reproduction in any medium, provided you give appropriate credit to the original author(s) and the source, provide a link to the Creative Commons license, and indicate if changes were made.

\section{References}

1. Cuello M, Ettenberg SA, Clark AS, Keane MM, Posner RH, Nau $\mathrm{MM}$, et al. Down-regulation of the erbB-2 receptor by trastuzumab enhances tumor necrosis factor related apoptosis inducing ligand mediated apoptosis in breast and ovarian cancer cell lines that overexpress erbB-2. Cancer Res. 2001;61:4892-900.

2. Bang YJ, Van Cutsem E, Feyereislova A, Chung HC, Shen L, Sawaki A, et al. Trastuzumab in combination with chemotherapy versus chemotherapy alone for treatment of HER2-positive advanced gastric or gastro-oesophageal junction cancer (ToGA): a phase 3, open-label, randomized controlled trial. Lancet. 2010;376:687-97.

3. Van Cutsem E, Bang YJ, Feng-yi F, Xu JM, Lee KW, Jiao SC, et al. HER2 screening data from ToGA: targeting HER2 in gastric and gastroesophageal junction cancer. Gastric Cancer. 2014. doi:10.1007/s10120-014-0402-y.

4. Bang YJ, Chung H, Xu J, Lordick F, Sawaki A, Lipatov O, et al. Pathological features of advanced gastric cancer (GC): relationship to human epidermal growth factor receptor 2 (HER2) positivity in the global screening programme of the ToGA trial. J Clin Oncol. 2009;27:4556.

5. Yonemura Y, Ninomiya I, Yamaguchi A, Fushida S, Kimura H, Ohoyama $\mathrm{S}$, et al. Evaluation of immunoreactivity for erbB-2 protein as a marker of poor short term prognosis in gastric cancer. Cancer Res. 1991;51:1034-8.

6. Uchino S, Tsuda H, Maruyama K, Kinoshita T, Sasako M, Saito $\mathrm{T}$, et al. Overexpression of c-erbB-2 protein in gastric cancer. Cancer. 1993;2:3179-84.

7. Ooi A, Kobayashi M, Mai M, Nakanishi I. Amplification of c-erbB-2 in gastric cancer: detection in formalin-fixed, paraffinembedded tissue by fluorescence in situ hybridization. Lab Invest. 1998;78:345-51. 
8. Chen XZ, Zhang WH, Yao WQ, Liu JP, Zhou ZG, Chen ZX, et al. Immunohistochemical HER2 expression not associated with clinicopathological characteristics of stage I-III gastric cancer patients. Hepatogastroenterology. 2014;61:1817-21.

9. Shan L, Ying J, Lu N. HER2 expression and relevant clinicopathological features in gastric and gastroesophageal junction adenocarcinoma in a Chinese population. Diagn Pathol. 2013;8:76.

10. Son HS, Shin YM, Park KK, Seo KW, Yoon KY, Jang HK, et al. Correlation between HER2 overexpression and clinicopathological characteristics in gastric cancer patients who have undergone curative resection. J Gastric Cancer. 2014;14:180-6.

11. Pirrelli M, Caruso ML, Maggio MD, Armentano R, Valentini AM. Are biopsy specimens predictive of HER2 status in gastric cancer patients? Dig Dis Sci. 2013;58:397-404.

12. Watson S, Validire P, Cervera P, Zorkani N, Scriva A, Lemay F, et al. Combined HER2 analysis of biopsies and surgical specimens to optimize detection of trastuzumab-eligible patients in eso-gastric adenocarcinoma: a GERCOR study. Ann Oncol. 2013;24:3035-9.

13. Warneke VS, Behrens HM, Boger C, Becker T, Lordick F, Ebert MP, et al. Her2/neu testing in gastric cancer: evaluating the risk of sampling errors. Ann Oncol. 2013;24:725-33.

14. World Health Organization. Definition of an older or elderly person. http://www.who.int/healthinfo/survey/ageingdefnolder/en/.

15. Japanese Gastric Cancer Association. Japanese classification of gastric carcinoma: 3rd English edition. Gastric Cancer. 2011;14: 101-12.

16. Polkowski W, van Sandick J, Offehaus G, ten Kate FJ, Mulder J, Obertop $\mathrm{H}$, et al. Prognostic value of Lauren classification and c-erbB-2 oncogene overexpression in adenocarcinoma of the esophagus and gastroesophageal junction. Ann Surg Oncol. 1999;6:290-7.

17. Tanner M, Hollmén M, Junttila TT, Kapanen AI, Tommola S, Soini Y, et al. Amplification of HER-2 in gastric carcinoma: association with topoisomerase IIa gene amplification, intestinal type, poor prognosis and sensitivity to trastuzumab. Ann Oncol. 2005; 16:273-8.

18. Lee KE, Lee HJ, Kim YH, Yu HJ, Yang HK, Kim WH, et al. Prognostic significance of p53, nm23, PCNA and c-erbB-2 in gastric cancer. Jpn J Clin Oncol. 2003;33:173-9.

19. Grabsch H, Silvakumar S, Gray S, Gabbert HE, Müller W. HER2 expression in gastric cancer: rare, heterogeneous and of no prognostic value-conclusions from 924 cases of two independent serious. Cell Oncol. 2010;63:839-42.

20. Marx AH, Tharun L, Muth J, Dancau AM, Simon R, Yekebas E, et al. Her-2 amplification is highly homogeneous in gastric cancer. Hum Pathol. 2009;40:769-77.

21. Barros-Silva JD, Leitao D, Afonso L, Vieira J, Dinis-Ribeiro M, Fragoso M, et al. Association of ERBB2 gene status with histopathological parameters and disease-specific survival in gastric carcinoma patients. Br J Cancer. 2009;100:487-93.

22. Matsubara J, Yamada Y, Hirashima Y, Takahari D, Okita N, Takashima A, et al. Impact of insulin-like growth factor type 1 receptor, epidermal growth factor receptor, and HER2 expressions on outcomes of patients with gastric cancer. Clin Cancer Res. 2008;14:3022-9.

23. Sheng WQ, Huang D, Ying JM, Lu N, Wu HM, Liu YH, et al. HER2 status in gastric cancers: a retrospective analysis from four Chinese representative clinical centers and assessment of its prognostic significance. Ann Oncol. 2013;24:2360-4.

24. Huang D, Lu N, Fan Q, Sheng W, Bu H, Jin X, et al. HER2 status in gastric and gastroesophageal junction cancer assessed by local and central laboratories: Chinese results of the HER-EAGLE study. PLoS One. 2013;8:1-7.

25. Esoki Y, Hirayama R, Hirokawa K. A comparison of patterns of metastasis in gastric cancer by histologic type and age. Cancer. 1990;65:2086-90.

26. Zheng H, Takahashi H, Murai Y, Cui Z, Nomoto K, Miwa S, et al. Pathobiological characteristics of intestinal and diffuse-type gastric carcinoma in Japan: an immunostaining study on the tissue microarray. J Clin Pathol. 2007;60:273-7.

27. Wu MS, Yang KC, Shun CT, Hsiao TJ, Lin CC, Wang HP, et al. Distinct clinicopathological characteristics of diffuse- and intestinal-type gastric cancer in Taiwan. J Clin Gastroenterol. 1997;25:646-9.

28. Yamashita K, Sakuramoto S, Katada N, Futawatari N, Moriya H, Hirai K, et al. Diffuse type advanced gastric cancer showing dismal prognosis is characterized by deeper invasion and emerging peritoneal cancer cell: the latest comparative study to intestinal advanced gastric cancer. Hepatogastroenterology. 2009;56:276-81.

29. Sauter G, Lee J, Bartlett JMS, Slamon DJ, Press MF. Guidelines for human epidermal growth factor receptor 2 testing: biologic and methodological considerations. J Clin Oncol. 2009;27: 1323-33. 